\title{
Increased Cardiovascular Risk Associated with Reduced Kidney Function
}

\author{
Timothy P. Ryan $^{a}$ Susan G. Fisher ${ }^{a}$ Jessica L. Elder ${ }^{a}$ Paul C. Winters ${ }^{a}$ \\ William Beckett ${ }^{\mathrm{b}}$ James Tacci ${ }^{\mathrm{a}}$ James A. Sloand ${ }^{\mathrm{c}}$ \\ ${ }^{a}$ Division of Epidemiology, Department of Community and Preventive Medicine, ${ }^{\mathrm{b}}$ Department of Environmental \\ Medicine, and ' Division of Nephrology, Department of Medicine, University of Rochester School of Medicine and \\ Dentistry, Rochester, N.Y., USA
}

\section{Key Words}

Chronic kidney disease • Glomerular filtration rate •

Cardiovascular mortality

\begin{abstract}
Background: Individuals with chronic kidney disease (CKD) are at substantial risk for cardiovascular mortality, but the risk associated with specific glomerular filtration rates (GFRs) is unknown. The objective of this study was to investigate the relationship between level of kidney function and the risk of cardiovascular mortality in a diverse population. Methods and Results: This was a nonconcurrent cohort study of 34,982 ambulatory patients. Kidney function was entered into the model as a time-dependent variable to minimize misclassification and allow for improved estimate of the effect of decreasing GFR on cardiovascular mortality. The adjusted hazard ratio for cardiovascular mortality was 1.00 (95\% Cl 0.93-1.06) with an estimated GFR (eGFR) of 45$59 ; 1.77(95 \% \mathrm{Cl} 1.65-1.89)$ with an eGFR $30-44 ; 3.75(95 \% \mathrm{Cl}$ 3.47-4.06) with an eGFR 15-29, and 3.83 (95\% Cl 3.40-4.33) with an eGFR $<15$. Conclusion: We demonstrate a graded risk of cardiovascular mortality with decreasing GFR, with a marked increase with an eGFR $<45 \mathrm{ml} / \mathrm{min} / 1.73 \mathrm{~m}^{2}$. These data also suggest that the availability of eGFR to physicians has had little impact on reducing the cardiovascular risk facing individuals with CKD. Our findings further highlight the
\end{abstract}

public health significance of CKD and the importance of its early identification and management to reduce cardiovascular mortality.

Copyright $\odot 2009$ S. Karger AG, Basel

\section{Introduction}

In 1974, Lindner et al. [1] first pointed out that patients treated by chronic renal replacement therapy are exposed to cardiovascular problems and suffer from accelerated and severe atheromatosis. Despite the decline in cardiovascular disease (CVD) in the general population over the past three decades, it remains the number one killer among those with end-stage renal disease (ESRD) [2]. Although we are only beginning to understand the relationship between CVD and chronic kidney disease (CKD), it is known that the cardiovascular mortality rate in ESRD patients is $\sim 10-20$ times that of the general population [3]. Moreover, those patients reaching ESRD without cardiovascular abnormalities have a high probability of developing subsequent new-onset CVD [4].

The process of cardiovascular damage starts very early in well-defined CKD patients, long before ESRD is reached [2]. Individuals in early stages of CKD (stages 1-4) are more likely to die from a cardiovascular event than to develop ESRD even after adjustment for other known risk 
factors [5, 6]. CVD has been shown to be twice as common in those with CKD as in the general population, with advancement at twice the rate [7]. In 1998, the National Kidney Foundation Task Force on Cardiovascular Disease in Chronic Renal Disease recommended that patients with CKD be considered in the 'highest risk group' for subsequent CVD events [2]. The increased risk of CVD is due, in part, to a higher prevalence of conditions recognized as conventional risk factors for CVD in the general population such as older age, hypertension, diabetes, physical inactivity, and hyperlipidemia [2]. Hemodynamic and metabolic factors characteristic of CKD, including anemia, proteinuria, increased extracellular fluid volume, and electrolyte imbalance may also contribute to the elevated risk [2]. Irrespective of cause, the presence of CKD remains an important independent risk factor for CVD. Numerous studies have examined the association of different cutoff values of serum creatinine with risks of death from cardiovascular causes and death from any cause. However, studies thus far have been limited by small sample sizes [8-12], the use of serum creatinine alone to determine kidney function, nonuniform cutoff values to define kidney disease $[8-11,13]$, and lack of information on longitudinal changes in estimated glomerular filtration rate (eGFR) and coexisting conditions [6, 8-13]. While Go et al. [6] demonstrated that reduced kidney function is an independent risk factor for mortality using single estimates of retrospectively calculated GFR based on a lastvalue-carried-forward method, our findings examine the relationship further by using serial estimates of kidney function per individual, which minimizes misclassification and allows for improved estimate of the effect of decreasing GFR on cardiovascular mortality for the entire cohort. In addition, unlike other studies, we did not retrospectively calculate GFR and were thus able to observe the impact that the availability of eGFR to physicians would have on patient outcomes.

The purpose of this study was to quantify the risk of death due to a cardiovascular event among individuals with CKD based on multiple estimates of kidney function.

\section{Methods and Materials}

\section{Study Population}

The study population was composed of individuals with and without CKD as defined by an eGFR $<$ or $\geq 60 \mathrm{ml} / \mathrm{min} / 1.73 \mathrm{~m}^{2}$, as reported in the laboratory records at Strong Memorial Hospital at the University of Rochester. Strong Memorial Hospital laboratories process specimens for both inpatient and ambulatory pa- tients and comprise roughly one third of all tests done in Monroe County, N.Y., USA. Given the potential for renal insult and instability, inpatient estimates were not included in the study and only ambulatory specimens were used in the analysis. The ambulatory patient specimens were collected from primary care offices throughout Monroe County. Laboratory data were obtained on the eligible study population of those 18 years or older, having at least one outpatient determination of serum creatinine between June 1,2003, and May 31,2004, yielding a sample of 82,976 unique patients. It was determined for this population that approximately $21 \%(17,827)$ had CKD as defined by an eGFR $<60 \mathrm{ml} / \mathrm{min} /$ $1.73 \mathrm{~m}^{2}$. Although the eligibility criterion was a single eGFR $<60$ $\mathrm{ml} / \mathrm{min} / 1.73 \mathrm{~m}^{2}, 78 \%$ of those defined as having CKD met the National Kidney Foundation definition of CKD (GFR $<60 \mathrm{ml} /$ $\mathrm{min} / 1.73 \mathrm{~m}^{2}$ of body surface area for $\geq 3$ months) [14].

This nonconcurrent cohort study examined the association between severity of CKD and risk of cardiovascular mortality. All subjects were followed for a maximum of 2.5 years (June 1, 2003, through December 31, 2005). The cohort was categorized into those with an eGFR $<60$ or $\geq 60 \mathrm{ml} / \mathrm{min} / 1.73 \mathrm{~m}^{2}$ using the Modification of Diet in Renal Disease (MDRD) equation reported by the laboratory between June 1, 2003, and May 31, 2004. Serum creatinine was measured using an enzymatic method (Vitros analyzer; Ortho-Clinical Diagnostics, Rochester, N.Y., USA). The eGFR was determined using the following four-variable MDRD equation: $\mathrm{eGFR}=175 \cdot 1(-1.154) \cdot 18(-0.203) \cdot(0.742$ if female) $\cdot(1.210$ if African-American) [15]. Serum creatinine is measured in $\mathrm{mg} / \mathrm{dl}$, and age in years. Calibration of the study analyzer has been described elsewhere [16]. Given the preidentified group of 17,827 individuals with an eGFR $<60 \mathrm{ml} / \mathrm{min} / 1.73 \mathrm{~m}^{2}$, we randomly selected a sample of 17,827 individuals with an $\mathrm{eGFR} \geq 60 \mathrm{ml} / \mathrm{min} / 1.73 \mathrm{~m}^{2}$ from the study population for use as an internal control group.

The first measurement of eGFR during the study period was considered the subject's index date (baseline). Follow-up time was defined as the period of entry into the study (first eGFR) to the date of event (mortality), or to the end of the study. Individuals who were not deceased prior to January 1, 2006, were defined as administratively censored. For those individuals who expired during the study, time to event was first eGFR to date of death. We excluded individuals who had already received a kidney transplant and those who were receiving dialysis at entry. Individuals who initiated dialysis or received a transplant during follow-up were censored on the date of transplant or initiation of dialysis. There were 623 individuals excluded from the study due to missing demographic data, missing social security number or emigration from New York State. The study was approved by the Institutional Review Board at the University of Rochester Medical Center in accordance with Health Insurance Portability and Accountability Act guidelines.

Outcomes of Interest

The primary outcome of interest was death from cardiovascular cause. A cardiovascular cause, as defined by ICD-10 codes, includes Myocardial (I11-I14), Coronary (I20-I51), Cerebrovascular (I60-I69), Peripheral Vascular (I70, I73-I75) and Nonspecific (Y40-Y45, Y49, Y50, T823, T825, T828, T850, Z951, Z955). Cardiovascular death was determined from the death certificates as inclusion in the primary, secondary, or tertiary cause of death. To ascertain the outcome status of the cohort, deaths were identi- 
fied through the United States Social Security Administration (USSSA) for the 2.5-year period. We provided the USSSA with the names and social security numbers of subjects in the cohort. The USSSA then provided the overall status (deceased or alive) of each individual. Death certificates were obtained from the New York State Department of Vital Statistics. Comorbid conditions used in the analysis (anemia, CVD, diabetes and hypertension) were obtained from the Strong Health ambulatory database and were based on two or more physician-assigned diagnoses, as indicated by ICD-9 codes.

\section{Statistical Analysis}

Descriptive analyses include an examination of the main exposure of interest, CKD. Multivariate analyses were stratified by the following GFR categories $(<15,15-29,30-44,45-59,60 \mathrm{ml} /$ $\left.\min / 1.73 \mathrm{~m}^{2}\right) . \chi^{2}$ tests were performed to compare categorical variables; $t$ tests were conducted for identification of differences between groups in continuous variables. All reported $p$ values are two tailed, and significance was ascribed to $p$ values $<0.05$. Age, race and gender-specific rates of death were estimated. Standardized mortality ratios (SMRs) were calculated based on internal controls (eGFR $\geq 60 \mathrm{ml} / \mathrm{min} / 1.73 \mathrm{~m}^{2}$ ) and the New York State population. Standardization was based on age, race and gender.

Kaplan-Meier survival analysis was used to estimate the nonparametric survival distribution among study participants by exposure (CKD vs. No CKD), eGFR strata, age group, gender, race, anemia, CVD, diabetes, and hypertension. For all survival analyses, follow-up time was defined as the period of entry into the study (first eGFR) to the date of event (mortality), or to the end of the study. To evaluate the independent effect of eGFR on outcomes, Cox proportional hazards models were used. Estimated GFR was entered into the model as a time-dependent variable to minimize misclassification and allow for improved estimate of the effect of decreasing GFR on cardiovascular mortality. The available variables known to be associated with GFR (gender, race, and age) were included in the model. Additional risk factors entered in the model included the presence of anemia, CVD, diabetes, and hypertension. Definition of comorbid conditions was based on two or more physician-assigned diagnoses, as indicated by ICD-9 codes in the ambulatory database. The group of subjects with an eGFR $\geq 60 \mathrm{ml} / \mathrm{min} / 1.73 \mathrm{~m}^{2}$ was used as the reference group in the analysis of the association between the level of the eGFR and cardiovascular outcome. The proportionality assumption of Cox models was assessed by including an interaction term in the model consisting of the predictor and the log of time and inspection of the complementary $\log [-\log$ (survival function)] curves. The proportional hazards assumption was not violated. All analyses were conducted using SAS software (version 9.1).

\section{Results}

The demographic characteristics of the cohort are outlined in table 1 . The study participants with CKD were on average 18 years older, were more likely to be female and Caucasian, and had a greater prevalence of anemia, diabetes, CVD and hypertension than those without $\mathrm{CKD}$. The mean $( \pm \mathrm{SD})$ number of serum creatinine mea- surements and mean interval between measurements for the cohort were $6.1 \pm 8.5$ and $3.6 \pm 3.3$ months, respectively. The median time of follow-up was 2.1 years. There were 1,171 (3.35\%; 95 CI 3.16-3.54\%) cardiovascular deaths identified among the 34,982 individuals in the cohort. The risks and rates of cardiovascular mortality among those with and without CKD are outlined in table 2. There were $1,005(5.8 \%)$ cardiovascular deaths among the 17,463 individuals with CKD. In contrast, there were $166(0.95 \%)$ cardiovascular deaths among the 17,519 individuals without CKD. The rate of cardiovascular mortality among those with and without CKD was 2.4 and 0.38 per 1,000 person-months, respectively. The overall risk of cardiovascular death was 57.6 per 1,000 persons among those with CKD and 9.5 per 1,000 persons among those without CKD. Cardiovascular-specific SMRs for those with and without CKD are provided in table 2. Cardiovascular mortality among those with CKD was higher (1.91; 95\% CI 1.79-2.03\%) than either of those without CKD or the New York State population rates (1.45; 95\% CI 1.36-1.54\%). The cardiovascular mortality rate among those without CKD was lower $(0.87 ; 95 \%$ CI $0.74-1.02 \%)$ than the New York State population rates used for comparison.

Population survival was estimated using the KaplanMeier method. Figure 1 illustrates survival during the course of follow-up stratified by eGFR. The difference between the eGFR strata was statistically significant $(\mathrm{p}<$ 0.0001 ). The 1-year survival for eGFR stratum was as follows: eGFR $<15$ (91.7\%), eGFR 15-29 (91.7\%), eGFR 30-44 (95.8\%), eGFR 45-59 (97.7\%), and eGFR $\geq 60$ (99.5\%).

After adjustment for differences in demographic characteristics and the presence or absence of anemia, CVD, diabetes, and hypertension, the risk of cardiovascular mortality increased sharply as eGFR decreased. The associated adjusted risk of cardiovascular mortality for each eGFR stratum was as follows: 1.00 (95\% CI $0.93-$ $1.06 \%$ ) for those with eGFR 45-59; 1.77 (95\% CI 1.65$1.89 \%$ ) for eGFR 30-44; 3.75 (95\% CI 3.47-4.06\%) for those with eGFR $15-29$, and 3.83 (95\% CI $3.40-4.33 \%$ ) for those with an eGFR $<15$. The associated risks of cardiovascular mortality for CVD, diabetes, and anemia were $2.06,1.48$, and 1.98 , respectively. With the exception of race (Black and other), all variables in the model were statistically significant. In the model, female gender and the presence of hypertension suggest a protective effect for cardiovascular mortality risk.

To further explore the finding of the apparent protective effect of hypertension, we conducted a survival anal- 
Fig. 1. Kaplan-Meier survival curves for cardiovascular mortality by estimated GFR strata. The difference in survival was statistically significant $(\mathrm{p}<0.0001)$. GFRs are measured in $\mathrm{ml} / \mathrm{min} / 1.73 \mathrm{~m}^{2}$. The Kaplan-Meier survival curves are not adjusted.

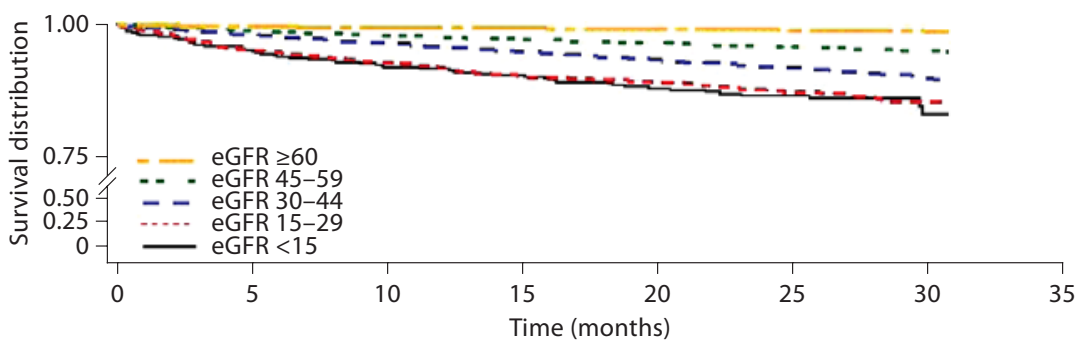

Table 1. Cohort with and without CKD

\begin{tabular}{|c|c|c|c|}
\hline & $\begin{array}{l}\text { CKD present } \\
(\mathrm{n}=17,463)\end{array}$ & $\begin{array}{l}\text { CKD absent } \\
(\mathrm{n}=17,519)\end{array}$ & $\begin{array}{l}\mathrm{p} \\
\text { value }\end{array}$ \\
\hline MDRD eGFR & $49.27 \pm 19.32$ & $85.48 \pm 23.50$ & $<0.001$ \\
\hline \multicolumn{4}{|l|}{ eGFR } \\
\hline$<15$ & $467(2.7)$ & & \multirow[t]{4}{*}{$<0.001$} \\
\hline $15-29$ & $1,162(6.7)$ & & \\
\hline $30-44$ & $3,839(21.9)$ & & \\
\hline $45-59$ & $11,995(68.7)$ & & \\
\hline$\geq 60$ & $0(0.00)$ & $17,519(100.0)$ & \\
\hline Age, years & $70.25 \pm 14.00$ & $52.49 \pm 15.61$ & $<0.001$ \\
\hline \multicolumn{4}{|l|}{ Age group } \\
\hline $18-49$ years & $1,652(9.5)$ & $7,527(42.9)$ & \multirow[t]{5}{*}{$<0.001$} \\
\hline $50-59$ years & $2,054(11.8)$ & $4,290(24.5)$ & \\
\hline 60-69 years & $3,766(21.6)$ & $3,087(17.6)$ & \\
\hline $70-79$ years & $5,208(29.8)$ & $1,774(10.1)$ & \\
\hline $80+$ years & $4,783(27.4)$ & $841(4.8)$ & \\
\hline \multicolumn{4}{|l|}{ Race } \\
\hline Caucasian & $15,422(88.3)$ & $14,133(80.7)$ & \multirow[t]{5}{*}{$<0.001$} \\
\hline African-American & $1,610(9.2)$ & $2,556(14.6)$ & \\
\hline Hispanic & $292(1.7)$ & $538(3.1)$ & \\
\hline Asian & $127(0.7)$ & $278(1.6)$ & \\
\hline Native American & $12(0.07)$ & $14(0.08)$ & \\
\hline \multicolumn{4}{|l|}{ Gender } \\
\hline Female & $10,844(62.1)$ & $9,577(54.7)$ & \multirow[t]{2}{*}{$<0.001$} \\
\hline Male & $6,619(37.9)$ & $7,942(45.3)$ & \\
\hline Hypertension & $13,238(75.8)$ & $6,777(38.7)$ & $<0.001$ \\
\hline Anemia & $7,268(41.6)$ & $2,441(13.9)$ & $<0.001$ \\
\hline Diabetes & $6,325(36.2)$ & $3,087(17.6)$ & $<0.001$ \\
\hline CVD & $12,467(71.4)$ & $6,443(36.8)$ & $<0.001$ \\
\hline \multicolumn{4}{|c|}{$\begin{array}{l}\text { Values are expressed as mean } \pm \text { standard deviation for age } \\
\text { and MDRD-estimated GFR. Otherwise, values expressed as } \mathrm{n} \\
\text { (\%). Differences between groups for categorical and continuous } \\
\text { variables were examined using } \chi^{2} \text { tests or Student's t tests, respec- } \\
\text { tively. MDRD-estimated GFRs are measured in } \mathrm{ml} / \mathrm{min} / 1.73 \mathrm{~m}^{2} \text {. } \\
\text { Comorbid conditions (hypertension, anemia, diabetes, and CVD) } \\
\text { are based on ICD-9 diagnosis codes identified through Strong } \\
\text { Health medical records. }\end{array}$} \\
\hline
\end{tabular}

Table 2. Risk of cardiovascular death

\begin{tabular}{|c|c|c|}
\hline & $\begin{array}{l}\text { CKD present } \\
(\mathrm{n}=17,463)\end{array}$ & $\begin{array}{l}\text { CKD absent } \\
(\mathrm{n}=17,519)\end{array}$ \\
\hline Observed deaths & 1,005 & 166 \\
\hline Expected deaths ${ }^{\mathrm{a}}$ & 526 & \\
\hline $\mathrm{SMR}^{\mathrm{a}}$ & 1.91 & \\
\hline $\mathrm{SMR}^{\mathrm{b}}$ & 1.45 & 0.87 \\
\hline Median time to death, months & 10.9 & 10.4 \\
\hline Person-years & 34,578 & 36,318 \\
\hline Average follow-up, years & 2.1 & 2.1 \\
\hline Rate of death ${ }^{c}$ & 2.4 & 0.38 \\
\hline Risk of death ${ }^{\mathrm{d}}$ & 57.6 & 9.5 \\
\hline \multicolumn{3}{|l|}{ Age-specific risk $^{\mathrm{d}}$} \\
\hline $18-49$ years & 15.1 & 1.6 \\
\hline $50-59$ years & 27.8 & 5.8 \\
\hline $60-69$ years & 23.6 & 7.5 \\
\hline $70-79$ years & 44.5 & 19.7 \\
\hline $80+$ years & 125.9 & 84.4 \\
\hline \multicolumn{3}{|l|}{ Race-specific risk ${ }^{\mathrm{d}}$} \\
\hline Caucasian & 59.3 & 9.6 \\
\hline African-American & 44.1 & 9.0 \\
\hline Hispanic & 54.8 & 9.3 \\
\hline Asian & 31.4 & 7.2 \\
\hline Native American & 0.0 & 0.0 \\
\hline \multicolumn{3}{|l|}{ Gender-specific risk $^{\mathrm{d}}$} \\
\hline Female & 55.3 & 8.0 \\
\hline Male & 61.2 & 11.2 \\
\hline
\end{tabular}

SMRs were calculated based on internal controls (eGFR $>60$ $\mathrm{ml} / \mathrm{min} / 1.73 \mathrm{~m}^{2}$ ) and the New York State population. Standardization was based on age, race and gender.

${ }^{\text {a }}$ Compared to unexposed.

b Compared to New York death rates.

${ }^{c}$ Rate per 1,000 person-months.

${ }^{\mathrm{d}}$ Risk per 1,000 persons. 
ysis stratified by hypertension for each age group. The results revealed an apparent protective effect for cardiovascular mortality endpoint among the $>80$ years age group. Cox models were also run with eGFR, gender, and hypertension stratified by age group; a protective effect was noted for the $>80$ years age group. Rastas et al. [17] demonstrated that a systolic blood pressure $\geq 160 \mathrm{~mm}$ $\mathrm{Hg}$ in individuals aged 85 years and over has a protective effect for all-cause mortality. These authors speculate that low systolic blood pressure may be partially related to poor general health. This altered risk factor or 'reverse epidemiologic' pattern, observed between CKD and the presence of hypertension, has been noted by others and deserves further study [18].

\section{Discussion}

The relationship between CKD and increased risk of cardiovascular mortality is not fully understood. Within our cohort, we observed increased prevalence of CVD and other known risk factors among those with CKD; these conditions were also more prevalent among those who subsequently died from CVD. The increased risk of CVD is due, in part, to a higher prevalence of conditions recognized as conventional risk factors for CVD in the general population such as older age, hypertension, diabetes, physical inactivity, and hyperlipidemia [2]. The elevated risk may also be contributed by hemodynamic and metabolic factors characteristic of CKD, including anemia, proteinuria, increased extracellular fluid volume, and electrolyte imbalance [2].

There are important implications of this study. The first is that the availability and application of eGFR by physicians did not improve patient outcomes. Physicians in our study had a 'real-time' eGFR. Therefore, clinical decisions and treatment could be made prospectively based upon eGFR. Algorithms to estimate GFR were developed to provide physicians with a more accurate estimate of kidney function. This should have, in turn, allowed physicians to identify and better manage those with CKD. In the study by Go et al. [6], physicians did not have an eGFR, but were basing clinical decisions on serum creatinine. Despite the additional information in the form of an eGFR in our study, individual risk did not improve. Our findings were otherwise consistent with those of Go et al. [6], who demonstrated that reduced kidney function is an independent risk factor for cardiovascular mortality. The slight differences in risk observed between our study and the primary analysis of Go et al. [6] can be explained by the level of analysis. Our study, which utilized serial estimates of GFR for all individuals, resulted in slightly attenuated risk in certain GFR strata. However, this was also observed by Go et al. [6] in their subgroup analysis utilizing serial estimates.

One of the study limitations was inclusion of only those individuals who have had a serum creatinine determination (eGFR) during the study period. Given that CKD is typically asymptomatic during early stages, it is likely that those with a reduced GFR who did not use medical services would not be included in the study, and this therefore limits the generalizability of our study to health-seeking individuals.

The severity of comorbid medical conditions (e.g. CVD, diabetes, hypertension, and anemia) was not known. In addition, no information on family medical history, blood pressure, physical activity, body mass index, and alcohol or tobacco consumption was available. Although tobacco consumption is a known renal and cardiovascular risk factor, we have no reason to believe that tobacco use among those with CKD is concentrated in the lower GFR strata. Further, the prevalence of smoking has been shown to decrease with age, and given that the average age increased as GFR decreased, this would only reduce the likely prevalence of tobacco consumption [19]. Nevertheless, residual confounding is unlikely to explain the large effect estimates observed for most categories of reduced eGFR. Lipid values were not available and could be a source of uncontrolled confounding.

Although CKD in this study was defined as eGFR $<60$ $\mathrm{ml} / \mathrm{min} / 1.73 \mathrm{~m}^{2}$, stratifying on level of GFR minimized the potential for misclassification. In addition, all subjects had serial estimates of GFR which allowed for a more accurate estimate of the effect of decreasing GFR on cardiovascular mortality. Entering the serial estimates into the model as a time-dependent variable had a significant impact on the analysis, reducing the risk of death (cardiovascular and all cause) in each of the GFR categories. The median number of estimates of GFR for the cohort was four. The Kidney Disease Outcomes Quality Initiative guidelines define CKD as not only an eGFR $<60 \mathrm{ml} /$ $\mathrm{min} / 1.73 \mathrm{~m}^{2}$, but also by the presence of fixed microalbuminuria, irrespective of GFR [14]. The number of individuals with an eGFR $\geq 60 \mathrm{ml} / \mathrm{min} / 1.73 \mathrm{~m}^{2}$ and microalbuminuria is unknown. Consequently, we may have underestimated the prevalence of CKD among the reference group of patients with an eGFR $\geq 60 \mathrm{ml} / \mathrm{min} / 1.73 \mathrm{~m}^{2}$.

The use of death certificates as a data source for outcome status (cardiovascular event) may have been limiting because the information is based on physician discre- 
tion, and may not be evidence based. However, we have no evidence that there was any misclassification bias in the vital status ascertainment or death certification between the exposed and unexposed. Finally, since our study was conducted among individuals who had serum creatinine measured as part of health care in western New York, our results may not be generalizable to persons in other geographic regions.

Our study has several advantages, including a large, diverse, outpatient population receiving usual care, with the inclusion of individuals with a spectrum of kidney function. This allowed for a detailed evaluation of the association between the level of kidney function and outcome. In addition, all subjects had serial estimates of GFR allowing for a more accurate estimate of the effect of decreasing GFR on cardiovascular mortality. The size and demographic characteristics of our study cohort, and the fact that specimens were collected from primary care offices throughout the County, increased the likelihood of a representative sample. By limiting our study to outpatient serum creatinine values and the use of one laboratory for estimation of GFR, we increased our confidence in the reliability of eGFR values.

In conclusion, we demonstrate a graded risk of cardiovascular mortality with decreasing GFR, with a marked increase with an eGFR $<45 \mathrm{ml} / \mathrm{min} / 1.73 \mathrm{~m}^{2}$. These data also suggest that the availability of eGFR to physicians has had little impact on reducing the cardiovascular risk facing individuals with CKD. Our findings further highlight the public health significance of CKD and the importance of its early identification and management to reduce cardiovascular mortality.

\section{Acknowledgment}

This study was supported in part by National Heart, Lung, and Blood Institute, grant T32 HL-07937.

\section{References}

1 Lindner A, Charra B, Sherrard DJ, Scribner BH: Accelerated atherosclerosis in prolonged maintenance hemodialysis. N Engl J Med 1974;290:697-701.

2 Levey AS, Beto JA, Coronado BE, Eknoyan G, Foley RN, Kasiske BL, et al: Controlling the epidemic of cardiovascular disease in chronic renal disease: what do we know? What do we need to learn? Where do we go from here? National Kidney Foundation Task Force on Cardiovascular Disease. Am J Kidney Dis 1998;32:853-906.

- 3 Foley RN, Parfrey PS, Sarnak MJ: Clinical epidemiology of cardiovascular disease in chronic renal disease. Am J Kidney Dis 1998; 32(suppl 3):S112-S119.

-4 Parfrey PS, Foley RN, Harnett JD, Kent GM, Murray D, Barre PE: Outcome and risk factors of ischemic heart disease in chronic uremia. Kidney Int 1996;49:1428-1434.

5 Foley RN, Murray AM, Li S, Herzog CA, McBean AM, Eggers PW, et al: Chronic Kidney Disease and the Risk for Cardiovascular Disease, Renal Replacement, and Death in the United States Medicare Population, 1998 to 1999. J Am Soc Nephrol 2005;16:489-495.

-6 Go AS, Chertow GM, Fan D, McCulloch CE, Hsu CY: Chronic kidney disease and the risks of death, cardiovascular events, and hospitalization. N Engl J Med 2004;351: 1296-1305.
7 Collins AJ, Li S, Gilbertson DT, Liu J, Chen SC, Herzog CA: Chronic kidney disease and cardiovascular disease in the Medicare population. Kidney Int 2003;87(suppl):S24-S31.

8 Wannamethee SG, Shaper AG, Perry IJ: Serum creatinine concentration and risk of cardiovascular disease: a possible marker for increased risk of stroke. Stroke 1997;28:557563.

9 Culleton BF, Larson MG, Wilson PWF, Evans JC, Parfrey PS, Levy D: Cardiovascular disease and mortality in a community-based cohort with mild renal insufficiency. Kidney Int 1999;56:2214-2219.

10 Mann JFE, Gerstein HC, Pogue J, Bosch J, Yusuf S, for the HI: Renal insufficiency as a predictor of cardiovascular outcomes and the impact of ramipril: The HOPE Randomized Trial. Ann Intern Med 2001;134:629636.

11 Shlipak MG, Heidenreich PA, Noguchi H, Chertow GM, Browner WS, McClellan MB: Association of renal insufficiency with treatment and outcomes after myocardial infarction in elderly patients. Ann Intern Med 2002; 137:555-562.

12 Garg AX, Clark WF, Haynes RB, House AA: Moderate renal insufficiency and the risk of cardiovascular mortality: results from the NHANES I. Kidney Int 2002;61:1486-1494.

13 Drey N, Roderick P, Mullee M, Rogerson M A population-based study of the incidence and outcomes of diagnosed chronic kidney disease. Am J Kidney Dis 2003;42:677-684.
14 National Kidney Foundation: K/DOQI clinical practice guidelines for chronic kidney disease: evaluation, classification, and stratification. Am J Kidney Dis 2002;39(suppl 1): S1-S266.

15 Levey AS, Bosch JP, Lewis JB, Greene T, Rogers N, Roth D: A more accurate method to estimate glomerular filtration rate from serum creatinine: a new prediction equation. Modification of Diet in Renal Disease Study Group. Ann Intern Med 1999;130:461-470.

16 Ryan TP, Sloand JA, Winters PC, Corsetti JP, Fisher SG: Chronic kidney disease prevalence and rate of diagnosis. Am J Med 2007; 120:981-986.

17 Rastas S, Pirttila T, Viramo P, Verkkoniemi A, Halonen P, Juva K, et al: Association between blood pressure and survival over 9 years in a general population aged 85 and older. J Am Geriatr Soc 2006;54:912-918.

18 Kovesdy CP, Trivedi BK, Kalantar-Zadeh K, Anderson JE: Association of low blood pressure with increased mortality in patients with moderate to severe chronic kidney disease. Nephrol Dial Transplant 2006;21:12571262

19 McCullough PA, Li S, Jurkovitz CT, Stevens L, Collins AJ, Chen SC, et al: Chronic kidney disease, prevalence of premature cardiovascular disease, and relationship to short-term mortality. Am Heart J 2008;156:277-283. 